\title{
Diabetes na Doença Coronária: O Risco do Não Diagnóstico
}

\section{Diabetes in Coronary Disease: The Risk of Non-Diagnosis}

João Filipe RAPOSO $\square^{1,2}$

Acta Med Port 2017 Jun;30(6):429-430 - https://doi.org/10.20344/amp.9353

Palavras-chave: Diabetes Mellitus; Doença Coronária; Estado Pré-Diabético; Portugal

Keywords: Coronary Disease; Diabetes Mellitus; Portugal; Prediabetic State

A diabetes mellitus é hoje um grave problema de saúde pública que ameaça não só a sustentabilidade dos serviços nacionais de saúde, mas também o potencial desenvolvimento e crescimento económico de todos os países.

Em Portugal, e de acordo com os dados do Observatório Nacional da Diabetes da Sociedade Portuguesa de Diabetologia, calcula-se que em $2015,13,3 \%$ da população adulta (entre os 20 e os 79 anos de idade) tivesse diabetes e $27,4 \%$ da mesma população hiperglicemia intermédia (a 'pré-diabetes').

Embora se saiba que a prevalência das doenças auto-imunes, de que a diabetes tipo 1 é um exemplo, esteja a aumentar, é a diabetes tipo 2 a responsável principal por este panorama.

O modelo de sociedade que adotámos parece estar intimamente ligado a esta ameaça. A inatividade física que começa logo na infância, a oferta alimentar desadequada, o nosso modelo de ocupação do território, um urbanismo pouco amigo da saúde com redes de transportes públicos pouco atrativas são alguns dos determinantes a que podemos ainda associar o baixo nível de literacia (formal e de saúde), padrões de escola e trabalho desadequados e o isolamento social da população idosa.

Conseguimos muitos dos objetivos que procurámos ao longo da nossa história - temos acesso mais fácil aos alimentos e precisamos de gastar menos calorias para obtê-los. Tal como acontece com alguns fármacos, este sucesso foi acompanhado de efeitos adversos graves. A epidemia da diabetes está associada à epidemia da obesidade, doenças cardiovasculares e alguns tipos de doenças oncológicas entre muitas outras. Vivemos mais anos, mas mais doentes, i.e., com pior qualidade de vida.

A diabetes está indicada como causa de morte em 4,0\% dos óbitos em Portugal e está presente em 25,9\% da letalidade intra-hospitalar. A diabetes é hoje uma das principais causas de hemodiálise (33,9\% dos novos casos), cegueira, amputações não traumáticas (1250 em 2015), episódios agudos de doença coronária $(32,4 \%$ dos internamentos por enfarte agudo do miocárdio) e de acidentes vasculares cerebrais (presente em $29,5 \%$ dos internamentos). O Observatório Nacional da Diabetes calcula que os custos diretos associados ao tratamento da diabetes representem $10 \%$ da despesa de saúde em Portugal (cerca de 1\% do PIB) a que se associam custos indiretos (dos próprios, famílias, comunidade) muito maiores.

A ameaça é representada pelo crescimento continuado da prevalência da diabetes que leva a maiores consumos de recursos de saúde - mais consultas, mais exames complementares de diagnóstico, mais medicamentos, mais soluções tecnológicas, mais tratamentos de complicações agudas e tardias da diabetes. A inovação que recentemente tem sido introduzida nestas áreas é também um fator adicional que contribuiu para uma explosão dos custos associados.

A solução, difícil, passará por uma aposta firme e diferente nos diferentes níveis de prevenção:

- Na prevenção primária - necessitamos de assumir que este é um desafio que vai muito além das estruturas de saúde - este é o desafio da nossa sociedade. O "Desafio Gulbenkian: Não à Diabetes" é um excelente modelo de cooperação entre Ministério da Saúde, estruturas municipais, coletividades, farmácias, Associação Protectora dos Diabéticos de Portugal (APDP), e muitos outros parceiros e que responde ao "Todos temos um papel a desempenhar";

- Na prevenção secundária - a aposta no diagnóstico precoce e tratamento inicial intensivo permitirá travar a progressão para as complicações e escolher opções terapêuticas farmacológicas e tecnológicas mais custo-efetivas;

- Na prevenção terciária - a organização de redes integradas de rastreio, diagnóstico e tratamento de complicações será a solução que prestará os melhores cuidados a quem deles precisa com o foco na pessoa e não na compartimentação artificial de cuidados.

As soluções terão obrigatoriamente de ser globais envolvendo todos os potenciais parceiros.

O trabalho apresentado neste número ${ }^{1}$ levanta um contributo importante para a discussão dos métodos diagnósticos da diabetes.

Chamamos diabetes mellitus ao conjunto de doenças crónicas que tendo mecanismos fisiopatológicos diferentes

\footnotetext{
1. Diretor Clínico. APDP - Associação Protetora dos Diabéticos de Portugal. Lisboa. Portugal.

2. Professor Auxiliar Convidado. Nova Medical School. Lisboa. Portugal.

$\square$ Autor correspondente: João Filipe Raposo. filipe.raposo@apdp.pt

Recebido: 25 de junho de 2017 - Aceite: 26 de junho de 2017 | Copyright @ Ordem dos Médicos 2017
} 
têm uma característica comum que é a hiperglicemia. Sabemos que a hiperglicemia, se não devidamente controlada ao longo dos anos, está associada ao aparecimento de complicações microvasculares e às complicações macrovasculares (em associação com a dislipidemia, hipertensão arterial e tabagismo). Os valores de glicemia que se consideram patológicos têm sido alvo de discussão permanente nas últimas décadas. A glicemia é uma variável biológica de distribuição contínua e a definição de limiares diagnósticos resulta de um balanço entre sensibilidade e especificidade e da avaliação difícil da evolução temporal das complicações.

Durante grande parte do século $X X$, o diagnóstico da diabetes passava pela realização de uma prova de tolerância oral à glucose (PTGO) - com quantidades de glucose, tempos e limiares diferentes ao longo de diferentes décadas. Posteriormente, a procura de soluções mais custo-efetivas, levou à adoção do valor da glicemia em jejum como um critério diagnóstico fundamental mantendo-se a PTGO para casos duvidosos. A HbA1c (hemoglobina glicada) foi adotada como critério diagnóstico pela Organização Mundial de Saúde em 2011, dado que além de ser usada como medida do controlo metabólico das pessoas com diabetes, poderia trazer valor diagnóstico acrescentado à glicemia pontual em jejum ao refletir a exposição do organismo à hiperglicemia nos 90 dias anteriores à sua medição.

O rastreio da diabetes, i.e., a definição de grupos populacionais que presumivelmente têm uma maior probabilidade de ter diabetes, era tradicionalmente efetuada por glicemias em jejum ou glicemias ocasionais. A introdução do FINDRISC como ferramenta de rastreio teve um duplo objetivo: por um lado identifica uma população de risco aumentado que pode beneficiar de medidas estruturadas de prevenção primária e que também deverá ser referenciada para testes diagnósticos; por outro lado, e ao ser aplicado à população global, pode funcionar como uma ferramenta educativa, já que associa alguns comportamentos menos saudáveis a scores de risco mais elevados.

As pessoas com diabetes têm, como referido, um risco

\section{REFERÊNCIAS}

1. Ribeiro A, et al. Estratégia de rastreio da diabetes na doença arterial coronária. Acta Med Port. 2017;30:432-40.

2. Gardete-Correia L, Boavida JM, Raposo JF, Mesquita AC, Fona aumentado de doença cardiovascular que de resto já está associado às fases de hiperglicemia intermédia (a 'pré-diabetes'). Este risco ainda se acentua mais após um primeiro episódio de doença macrovascular.

Com o estudo PREVADIAB, calcula-se que na população portuguesa cerca de $43 \%$ da população com diabetes desconheça o seu diagnóstico. ${ }^{2}$

Atendendo às necessidades referidas de fazer o diagnóstico precoce de diabetes em termos de saúde pública em geral e do risco cardiovascular em especial, o objetivo primário deste trabalho reveste-se da maior importância.

É um trabalho metodologicamente robusto, que analisa uma amostra consecutiva de doentes submetidos a angioplastia por doença coronária. Os testes laboratoriais foram efetuados entre 4 a 5 meses após a intervenção o que afasta a interferência das eventuais hiperglicemias do stress.

O estudo PREVADIAB tinha descrito que a utilização da $\mathrm{HbA} 1 \mathrm{c}$ perdia cerca de $30 \%$ dos diagnósticos efetuados às 2 horas da PTGO na população global. Neste trabalho, os autores referem que tal acontece em cerca de $17 \%$ da população estudada.

A utilização do FINDRISC, nunca validado para este tipo de população, traduziu-se por uma melhor capacidade diagnóstica, mas insuficiente face ao diagnóstico por PTGO.

Chama-se a atenção que $27 \%$ da população com doença arterial coronária que sofreu um episódio agudo já tinha a diabetes diagnosticada e que esta prevalência subiu para $36,8 \%$ com a aplicação de novos testes diagnósticos. Analisando melhor todos os dados, verificamos que $70 \%$ da população anteriormente internada tinha diabetes ou 'pré-diabetes'.

Os dados aqui apresentados deverão constituir um ponto de partida para uma discussão entre as diferentes sociedades científicas interessadas e que relance, de novo, o papel diagnóstico da PTGO nesta população.

O risco vascular é demasiado elevado para ficarmos a aguardar!

C, Carvalho R, et al. First diabetes prevalence study in Portugal: PREVADIAB study. Diabet Med. 2010;27:879-81. 